\title{
A NUMERICAL METHOD OF FINDING A SUITABLE BONUS SCALE
}

\author{
Erkit Pesonen \\ Helsinki (Finland)
}

The bonus system used by Finnish insurers is as follows: Policies are divided into 4 bonus classes, $C_{1}, C_{2}, C_{3}, C_{4}$, each of them being initially placed in class $C_{1}$. After one claimless year, a policy placed in class $C_{h}$ is transferred to class $C_{h+1}(h \leq 3)$ from the beginning of the following year. After a claim year, each policy is entered back to class $C_{1}$. (Whether the number of claims is one or more does not play any role in this transition system.) From the initial premium, i.e. the premium in class $C_{1}$, a reduction of $p_{i}$, per cent is granted in class $C_{i}$. In numbers these percentages are as follows:

$$
\text { in } \begin{aligned}
C_{1} & p_{1}=0 \% \\
C_{2} & p_{2}=10 \% \\
C_{3} & p_{3}=30 \% \\
C_{4} & p_{4}=50 \%
\end{aligned}
$$

In present investigations there is a trend to replace this scale by another in which mere contingency would not cause too great a change in the premium and in which the ,test period" would be longer.

Let us suppose that we want to find a suitable percentage scale $p=\left(p_{1}, p_{2}, \ldots, p_{m}\right)$ for a bonus system $\left(C_{1}, C_{2}, \ldots, C_{m}\right)$ with a given transition mechanism according to which for each bonus class $C_{h}$ two bonus classes $C_{i}$ and $C_{j}$ are given to which the policy is transferred after one year from $C_{h}$ according as there have been claims or not.

In order to simplify the problem we disregard the running expences and suppose that the distribution function of the amount of one claim is independent of the claim and the policy, so that we can content ourselves with observing the number of the claims, and not the amount of the claim. We further suppose that the probability of the occurrence of a claim remains constant in each policy and that the number of claims in each policy is Poisson 
distributed with parameter $\lambda$. This parameter is distributed according to policies and agreed with distribution function $U(\lambda)$ the density function of which is denoted by $u(\lambda)$. We finally suppose that the so-called hunger for bonus, i.e. the tendency of leaving trifling claims unnotified to the company in order to preserve bonus reduction, does not occur in the system.

Because the steady flow of new policies to the group under observation will essentially influence the calculation, a further simplifying supposition will be made: If the number of policies in a certain year is $N, \alpha N$ new policies will come to the group from the beginning of the following year (to a bonus class defined in the system, e.g. class $C_{1}$ ). We suppose that the new policies will be distributed in accordance with distribution function $U(\lambda)$. We further suppose that $\alpha$ is a constant.

As a criterion for the correct scale $p$ either of the following can be taken into consideration:

r. the premium for a very long time per each policy $=$ the expectation value of the indemnities of this policy;

2. the premium per each bonus class $=$ the expectation value of the indemnities of this class.

The fulfilment of requirement $I$ evidently presupposes that normally $p_{m}=$ Ioo if there are policies in which $\lambda \ll E[\Lambda]$, supposing that $C_{m}$ is the class with the lowest premium. A bonus system fulfilling this requirement has been studied by Grenander [I].

Requirement 2 can be fulfilled at least theoretically. If, however, $m>\mathrm{r}$, vector $\mathrm{p}$ is not constant even if we make the above suppositions. We can, however, content ourselves with examining vector p asymptotically. Suppose that all policies are first placed in the bonus class of all new policies. Sufficiently many years having elapsed, there are policies in every bonus class. The function $u$ being known, the expectation values per year of the risk premiums in different bonus classes can subsequently be calculated according to Bayes' formula. Similarly the vector

$$
p^{t}=\left(p_{1}^{t}, p_{2}^{t}, \ldots, p_{m}^{t}\right),
$$

fulfilling requirement 2 after the year $t$, can also be calculated.

Letting $\mathrm{t}$ grow without limit, we get the limit vector

$$
p=\lim p^{t}
$$


which we can consider a suitable bonus scale, provided of course that there is a limit.

To facilitate notations we shall adopt the following symbols: Let

$$
z(j)=\left(i_{1}, i_{2}, \ldots i_{j}\right) \quad\left(i_{k}=\text { o or } \mathrm{I}\right)
$$

be the vector indicating the series of events encountered by the policy during the years $0, \mathrm{I}, \ldots, j-\mathrm{I}$ so that $i_{k}=\mathrm{I}$ if there have been no claims in the year $k-\mathrm{I}$, otherwise $i_{k}=0$. Further let

$$
i=\sum_{i=1}^{i} i_{k}
$$

The condition that $z(j) \varepsilon C_{h}$ from the beginning of the year $j$ depends solely on the transition mechanism and is to be found out beforehand with different values of $j$ and $z(j)$.

In order that Bayes' formula could be applied in practice to numerical calculations, the distribution functions of the sub-groups $z(j)$, arising in the process, must be such that they do not make the calculations too complicated.

Suppose that in a certain group $z(j)$ the density function is $u(\lambda \mid z(j))$. When, at the end of the year, the policies are divided into 2 groups according as there have been claims or not, we get the following conditional density functions by using such symbols in Thyrion's article [2] as are suitable here:

$$
\begin{aligned}
& u(\lambda \mid z(j), N=0)=e^{-\lambda} u(\lambda \mid z(j)) / P\{N=0 \mid z(j)\} \\
& u(\lambda \mid z(j), N>0)=\left(\mathrm{I}-e^{-\lambda}\right) u(\lambda \mid z(j)) /(\mathrm{I}-P\{N=0 \mid z(j)\})
\end{aligned}
$$

both of which we hope to be formally of the same type as the original function $u(\lambda \mid z(j))$, and consequently of the same type as the initial density function $u(\lambda)$ of the whole process.

A class of this kind of the $u$-functions with three parametres is obtained by selecting

$$
u(\lambda ; a, k, s)=\left(\Gamma^{\top}(k) w(a, k, s)\right)^{-1} \lambda^{k-1} \sum_{j=0}^{\infty} \frac{\Gamma(j+s)}{\Gamma(j+\mathrm{I}) \Gamma(s)} e^{-(a+j) \lambda},
$$

where $a, k>0$ and $s \leq 0$ and

$$
w(a, k, s)=\sum_{j=0}^{\infty}(a+j)^{-k} \frac{\Gamma(j+s)}{\Gamma(j+\mathrm{I}) \Gamma(s)} .
$$


Since

$$
\begin{aligned}
& w(a, k, s) P[N=0]=w(a+\mathrm{I}, k, s) \\
& w(a, k, s) E[\Lambda]=k w(a, k+\mathrm{I}, s) \\
& w(a, k, s) E\left[\Lambda^{2}\right]=k(k+\mathrm{I}) w(a, k+2, s),
\end{aligned}
$$

we can, in principle at least, also estimate the parametres $a, k, s$ by estimating the quantities $P[N=0], E[\Lambda]$ and $E\left[\Lambda^{2}\right]$ from the statistical material.

The initial density function being thus selected, the conditional density function of class $z(j)$ can be calculated in the following way:

Let

$$
u(\lambda \mid z(j))=u(\lambda ; a+i, k, s+i-j)
$$

$$
\begin{aligned}
& F(j, i)=w(a+i, k, s+i-j) \\
& G(j, i)=k w(a+i, k+\mathrm{I}, s+i-j),
\end{aligned}
$$

then, after $n$ years in class $C_{h}$, provided $\alpha=0$

$$
E\left[\Lambda \mid C_{h}\right]=\frac{\sum_{i=0}^{n} A_{i}(n, h) G(n, i)}{\sum_{i=0}^{n} A_{i}(n, h) F(n, i)}
$$

where the coefficients $A_{i}(n, h)$ are integers and depend solely on the transition mechanism, besides depending on $n$ and $h$. The calculation will be somewhat more complicated if $\alpha \neq 0$. Hence, after $n$ years, we get

$$
E\left[\Lambda \mid C_{h}\right]=\frac{\sum_{i=0}^{n} A_{i}(n, h) G(n, i)+\alpha \sum_{i=0}^{n-1} \sum_{i=0}^{j}(\mathrm{I}+\alpha)^{n-j-1} A_{i}(j, h) G(j, i)}{\sum_{i=0}^{n-1} A_{i}(n, h) F(n, i)+\alpha \sum_{i=0}^{n} \sum_{i=0}^{n}(\mathrm{I}+\alpha)^{n-j-1} A_{i}(j, h) F(j, \imath)} .
$$

Thus the vector $p^{n}$ is after $n$ years

$$
p^{n}=\left(p_{1}^{n}, p_{2}^{n}, \ldots, p_{m}^{n}\right)
$$


where

$$
\left(\text { Ioo }-p_{h}^{n}\right)=\frac{E\left[\Lambda \mid C_{h}\right]}{E\left[\Lambda \mid C_{1}\right]} \text { roo }
$$

when it has been normed by selecting $p_{1}^{n}=0$ as the percentage for class $C_{1}$.

In numerical calculations the following sample out of the statistical material dealing with private motor-cars registered in Helsinki was available:

\begin{tabular}{cc}
$N$ & $\begin{array}{c}\text { Policies with } N \text { claims } \\
\text { during one year } \\
\text { o }\end{array}$ \\
I & $505^{8}$ \\
2 & 403 \\
3 & 34 \\
4 & 2 \\
5 & 0 \\
\hline
\end{tabular}

\section{8}

The material being so small, the computing of three parametres does not seem convincing, especially as certain observations derived from different material showed as if the $\Gamma$-distribution $(s=0)$

$$
u(\lambda)=a^{k} \frac{\lambda^{k-1}}{\Gamma(k)} e^{-a \lambda}
$$

could be selected as the initial distribution.

By estimating the parameters $a$ and $k$ the vectors $p$ were first introduced into the present bonus system. In the calculation the constant $\alpha=0, I$ was used. In this case the vector $p$ converged very quickly and led into the following percentages

$$
\begin{aligned}
& p_{1}=0 \% \\
& p_{2}=12 \% \\
& p_{3}=22 \% \\
& p_{4}=52 \%
\end{aligned}
$$

which agree fairly well with the current scale $(0,10,30,50)$. It is interesting to compare the computed percentages with the actual frequencies observed in different bonus classes. In the above sample 
the actual frequencies were (if we denote the frequency of the bonus class $C_{1}$ by IOO)

$$
\begin{array}{lr}
C_{1} & 100 \\
C_{2} & 8 \mathrm{r} \\
C_{3} & 47 \\
C_{4} & 50
\end{array}
$$

which could lead into the bonus system $(0, I 9,53,50)$. The difference between these values and the percentages obtained by computation can partly be explained by the scarcity of material as the number of policies was very small in groups $C_{2}$ and $C_{3}$. Another factor which doubtless will cause difference is the hunger for bonus which is a very difficult factor to manage numerically. It is evident that many claims remain unnotified, especially in other classes than $C_{1}$, due to the hunger for bonus. Thus the frequency in high bonus classes can be supposed to be lower than the "theoretical" frequency. At the same time, however, the risk level in the highest classes is lowered because more and more weak risks enter them. It has been possible to make an approximate study of the influence of the hunger for bonus in the present bonus system because of the fact that there is only very little hunger for bonus in class $C_{1}$. By only taking sufficient claims ( $\geqslant 20000 \mathrm{Fmk}$ ) into consideration, the distribution functions of the amount of one claim in different bonus classes have first been deduced, and it has been observed that with a very great accuracy they are the same in each bonus class. Subsequently the hypothesis has been made that if all claims were included, identical distribution functions would be obtained were there no hunger for bonus. According to this hypothesis, the influence of the hunger for bonus on the number and amount of claims can directly be calculated by examining the actual distribution functions. In the case of the private cars in Helsinki, the result was that in the highest bonus class 12 per cent of all claims were left unnotified, whereas the amount of indemnity they represented was only 2 per cent of the actual amount in the class in question.

The numerical calculation was also applied to the bonus system $\left(C_{1}, C_{2}, \ldots, C_{7}\right)$ with the following rules of transition:

I) new policies are placed in class $C_{1}$ 
2) after a claimless year the policy is transferred from class $C_{j}$ to class $C_{j_{+1}}$ (from class $C_{7}$ to class $C_{7}$ )

3) after a claim year the policy is transferred from class $C_{j}$ to class $C_{j_{-2}}$ (from classes $C_{2}$ and $C_{1}$ to class $C_{1}$ ).

The convergence of vector $p$ was now essentially slower. After I5 years the results will probably be correct with the accuracy of one per cent. The following results were obtained:

$$
\begin{aligned}
& p_{1}=0 \\
& p_{2}=9 \\
& p_{3}=14 \\
& p_{4}=19 \\
& p_{5}=22 \\
& p_{6}=29 \\
& p_{7}=55
\end{aligned}
$$

In this system the influence of the hunger for bonus can be expected to be smaller because the rise in the premium after a claim will remain smaller than before.

In conclusion we should like to assert that the above analysis must be accepted with reserve because the initial suppositions are very much simpler than reality. It was not possible to ascertain the consistency of the selection of the function $u(\lambda)$ with reality because of the scarcity of available material as even the ",allowed" deviation was so considerable that it can have a decisive effect on the final result. As there has, however, been a possibility of comparing the result obtained by this method with those obtained from the present bonus system it may be that by applying the method to new bonus systems one can nevertheless get the idea of correct bonus percentages, once the transition mechanism of the new system has been given.

\section{REFERENCES}

(I) Grenander, Ulf: Some Remarks on Bonus Systems in Automobile Insurance. Skand. Akt. Tidskrift 1957, pp. 180-197.

(2) Thyrion, P.: Contribution à l'étude du bonus pour non sinistre en assurance automobile, The ASTIN Bull., I, part III, pp. 142-162. 